\title{
Electroneurographic findings in patients with solvent induced central nervous system dysfunction
}

\author{
P ØRBÆK, ' I ROSÉN, ${ }^{2}$ K SVENSSON ${ }^{3}$ \\ From the Department of Occupational Medicine, ${ }^{\prime}$ Malmö General Hospital, Mälmo, and Department of Clinical \\ Neurophysiology, ${ }^{2}$ Lund University Hospital, and Department of Statistics, ${ }^{3}$ Lund University, Lund, Sweden
}

ABSTRACT The function of the peripheral nervous system was examined in a group of 32 men aged 30-65 (mean 49) with diagnosed solvent induced chronic toxic encephalopathy. The subjects were examined at the time of diagnosis and 26 were re-examined after a follow up period of 22-72 months (mean 40) and compared with a group of 50 unexposed male workers aged 27-64 (mean 42) with appropriate adjustment for age. All subjects were carefully scrutinised for alcohol abuse and other neurological diseases. The results of motor fibre neurography disclosed no difference between the groups. Nevertheless, a significant decrease in motor conduction velocity was found in the patients at follow up. Sensory fibre neurography showed signs of slight axonal degeneration with significantly decreased sensory nerve action potential amplitudes in the median and sural nerves; these amplitudes increased during follow up. The duration of sensory nerve action potentials was longer in the exposed group for the median and the sural nerves. The percentage of late components was significantly higher in the median nerve. The warm-cold sensitivity in the exposed group also indicated a slight sensory dysfunction with statistically significant wider detection limits.

Peripheral nerve damage is caused by some solvents including carbon disulphide, ${ }^{1}$ acrylamide, ${ }^{2}$ and $n$-hexane and its metabolites. ${ }^{3}$ Cross sectional studies on workers exposed to mixtures of solvents have shown slower conduction velocities ${ }^{45}$ and reduced action potentials ${ }^{5}$ among the exposed subjects. Knave et al found increased conduction velocities and reduced action potentials in workers exposed to jet fuel. ${ }^{6} \mathrm{We}$ have previously found similar changes with reduced action potential of the sural nerve in workers exposed to styrene and in a group of paint industry workers. ${ }^{78}$ The latter also tended to have increased conduction velocities. $^{8}$

The time course of toxic peripheral neuropathy in workers exposed to n-hexane or methyl n-butyl ketone has been described as a deterioration for several months after the cessation of exposure followed by recovery. ${ }^{910}$ Seppälainen and Antti-Poika described the time course of neurographic findings in 73 subjects with a diagnosis of "chronic solvent intoxication" based on pathological results in either psychometric or neurophysiological examinations." They found an increase of abnormal findings at a re-examination after three to nine years.

The aims of the present study are to describe the

Accepted 6 April 1987 functional status and the time course of possible changes in the peripheral nervous system (PNS) among subjects diagnosed to have central nervous system dysfunction. Methods were chosen with the objective of showing subclinical evidence of PNS dysfunction. ${ }^{1213}$

\section{Subjects}

The study group comprised 32 men aged 30-65 (mean 49) when they were given the diagnosis of chronic toxic encephalopathy. The diagnosis was based on symptoms and on psychometric test results. Electroneurographic examination was part of a complete evaluation of the nervous system. The results, however, have not been used for selection purposes.

The selection of subjects, the differential diagnostic examination, exposure, and the results of computed brain tomography and EEG are described in detail elsewhere. ${ }^{14}$ is Regional cerebral blood flow (rCBF) has also been measured on these subjects. ${ }^{\text {15a }}$ Alcohol consumption was less than $200 \mathrm{~g} / \mathrm{w}$ for 26 subjects and less than $400 \mathrm{~g} / \mathrm{w}$ for the remaining six.

None of the subjects suffered from diseases known to cause neuropathy. Twenty one had complaints of paraesthesias at follow up. The clinical examination disclosed signs of mild sensory neuropathy in 
eight subjects and definite neuropathy in another eight. ${ }^{15 b}$ The clinical classification was based on reduced sensation for touch or needle prick on the feet and distally on the legs or both. The eight subjects who had signs of mild sensory disturbance all had normal spinal reflexes; five were under 50. Of the eight with more pronounced sensory disturbance, reduced spinal reflexes on the legs were found in six and in two the reflexes were absent. In the arms reflexes were absent in one, reduced in five, and normal in two subjects. These eight subjects with polyneuropathy were over 50 .

A re-examination was made $22-72$ months (mean 40) after the diagnosis of encephalopathy when the subjects had not been exposed to solvents for 3-93 months (mean 36). Six subjects have not been reexamined due to an administrative mistake. They all had normal results at the first examination when their ages were $34,34,37,37,52$, and 60 .

An additional 11 subjects had previously been reexamined but refused to take part in the complete differential diagnostic evaluation and are thus not included in the results presented in this paper. The follow up results were considered in two subgroups divided at the age of 50 . Subgroup "young" comprised 11 subjects aged 32-48 (mean 38 ) and subgroup "old" 15 subjects aged $51-65$ (mean 58 ). Exposure free time and time between the two examinations did not differ significantly in the two subgroups. Exposure is described in more detail in our paper concerning the EEG power spectrum analysis of the group. ${ }^{14}$ The exposure index was significantly higher in subgroup old (mean $62 \vee 30 \mathrm{p}<0.001$ ). None of the subjects had, to our knowledge, been exposed to substances known to cause peripheral neuropathy. ${ }^{3}$

The control group consisted of 50 men aged 27-64 (mean 42) from the same socioeconomic level as the study group. They had never been occupationally exposed to solvents and all were in good health. ${ }^{8}$ Alcohol consumption was less than $200 \mathrm{~g} / \mathrm{w}$ for 44 subjects and less than $400 \mathrm{~g} / \mathrm{w}$ for the remaining six.

\section{Methods}

A Disa 1500 equipment with a constant current stimulator was used. The subjects were warmed if necessary to obtain skin surface temperatures exceeding $30^{\circ} \mathrm{C}$ in the hands and feet.

Conduction velocities of the fast motor fibres were determined for the median, ulnar, peroneal, and posterior tibial nerves. Compound muscle action potentials were recorded with surface electrodes from the thenar, hypothenar, extensor digitorum brevis, and abductor hallucis muscles. The nerves were stimulated at the wrist, elbow, ankle, and knee. The active recording electrode was placed so that the initial part of the muscle action potential showed a mono- phasic negativity. Distal latencies, defined as the latencies from the distal stimulus to the onset of the M-wave, were also measured.

Sensory neurography was performed with surface electrodes on the median and sural nerves. Fingers I and III were stimulated by ring electrodes and the sensory action potentials were recorded over the median nerve immediately proximal to the wrist (interelectrode distance $30 \mathrm{~mm}$ ). The stimulus current was adjusted to produce a maximal nerve volley (usually about $15 \mathrm{~mA}$ ). The sensory action potential of the median nerve was also recorded with a needle electrode positioned close to the nerve and one needle electrode placed $20 \mathrm{~mm}$ more laterally. With needle electrodes it is possible to define late components of the compound nerve action potential. The late components are quantified in proportion to the amplitude of the main nerve action potential complex. Sensory action potentials from the sural nerve were measured antidromically at the lateral malleolus when the nerve was stimulated at the posterolateral side of the calf. Latency, duration, amplitude, and late components were measured by averaging 128 individual records.

Thresholds to vibration at $100 \mathrm{~Hz}$ were determined at the carpus and pretibially using the method of Goldberg and Lindblom. ${ }^{16}$ Warm-cold difference limens were measured at the chin and at the thenar and lateral edge of the foot by the method of Fruhstorfer $e t$ al..$^{17}$ The rate of change in temperature was $2^{\circ} \mathrm{C} / \mathrm{s}$.

\section{DATA ANALYSIS}

Comparisons between groups were performed using a general linear model with adjustment for age. Comparisons within groups were performed using Student's paired $t$ test. Covariation between time variables and neurographic measures were investigated by correlation analysis. All $p$ values are two sided.

The analysis of missing data showed a random pattern of minor frequency. The frequency, however, was large enough to invalidate any multivariate approach to the data. Thus the results are shown variable by variable. When analysing a substantial number of variables one by one, the problem of mass significance must be considered and the pattern of significant results should be considered as a whole rather than single occurrences among the variables.

\section{Results}

Motor fibre neurography showed no clear differences between exposed and unexposed subjects (table 1). The exposed group had slower conduction velocities at follow up that could only partly be explained by increasing age. The regression coeffieients for age (year) in the control group were $-0.17,-0.16$, $-0 \cdot 18$, and $-0 \cdot 13$ respectively.

Distal motor latency was similar in the two groups 


\begin{tabular}{|c|c|c|c|c|c|c|}
\hline & \multirow{2}{*}{\multicolumn{2}{|c|}{ Controls $(n=50)$}} & \multicolumn{4}{|c|}{ Patients with toxic encephalopathy } \\
\hline & & & \multicolumn{2}{|c|}{ Diagnosis $(n=32)$} & \multicolumn{2}{|c|}{ Follow up $(n=26)$} \\
\hline & Mean & $S D$ & Mean & $S D$ & Mean & $S D$ \\
\hline \multicolumn{7}{|c|}{ Motor conduction velocity $\mathrm{m} / \mathrm{s}$ : } \\
\hline Median & $58 \cdot 7$ & $4 \cdot 6$ & $55 \cdot 8^{*}$ & $3 \cdot 4$ & $54 \cdot 2 \ddagger$ & $4 \cdot 5$ \\
\hline Ulnar & $57 \cdot 7$ & $5 \cdot 5$ & $56 \cdot 6$ & 4.9 & $55 \cdot 2^{+}$ & $5 \cdot 0$ \\
\hline Peroneal & $45 \cdot 8$ & $3 \cdot 7$ & $44 \cdot 8$ & $4 \cdot 3$ & $43 \cdot 8+$ & $4 \cdot 6$ \\
\hline Posterior tibial & $44 \cdot 7$ & $3 \cdot 5$ & $43 \cdot 0$ & $4 \cdot 6$ & $41 \cdot 9$ & $4 \cdot 6$ \\
\hline \multicolumn{7}{|c|}{ Distal motor latency ms: } \\
\hline Median & $3 \cdot 8$ & 0.4 & $4 \cdot 0$ & 0.4 & $3 \cdot 9$ & 0.5 \\
\hline Ulnar & $3 \cdot 1$ & $0 \cdot 3$ & $3 \cdot 1$ & $0 \cdot 3$ & $3 \cdot 1$ & 0.5 \\
\hline Peroneal & $4 \cdot 8$ & 0.8 & $4 \cdot 3^{*}$ & 0.7 & $4 \cdot 7 \ddagger$ & $1 \cdot 1$ \\
\hline Posterior tibial & $5 \cdot 9$ & $1 \cdot 3$ & $5 \cdot 9$ & $1 \cdot 3$ & $6 \cdot 0^{+}$ & 1.9 \\
\hline
\end{tabular}

Significance level for age adjusted group comparisons: ${ }^{*} \mathrm{p}<0.05$.

Significance levels in $t$ test for paired data in comparisons between the two examinations: $\nmid p<0.1 ; \neq p<0.05$.

although in one nerve, the peroneal, the exposed group had a significantly shorter latency $(p<0.01)$ that became longer at follow up.

Sensory conduction velocities were equal in the two groups (table 2). In the exposed group the velocities decreased more than expected from the age (year) coefficients $(-0 \cdot 1,-0 \cdot 2,-0 \cdot 1)$ during the approximate three years of follow up. Sensory nerve action potential amplitudes were smaller in the patient group. The differences were statistically significant in both the median $(p<0.05)$ and sural nerves $(p<0.01)$. The amplitudes increased during follow up despite an expected reduction with increasing age (age/year coefficients $-0.65,-0.43,-0.25$ ).

Sensory nerve action potential durations were longer in the exposed subjects (median $p<0 \cdot 1$, sural $p<$ 0.05 ) and there was no significant change at the follow up examination. The percentage of late components was increased in the median nerve $(p<0.01)$; there was no significant change at follow up.

The psychophysical test for large myelinated sensory fibres, the vibration threshold, gave equal results in the two groups. During follow up there was no change (table 3). For the warm-cold difference limens testing thinner myelinated and unmyelinated fibres we found reduced function among the exposed subjects (table 3). We found no change after the unexposed interval.

The exposure index was only significantly correlated with measures in which the patient group did not deviate from the controls. Only for the warm-cold difference limens hand and foot was there a suggestion of an exposure effect relation with $r=0.36(p<0.09)$ and $\mathrm{r}=0.38(\mathrm{p}<0.08)$ respectively.

Covariation between unexposed time and the difference between the examinations was also con-

Table 2 Results of sensory fibre neurography

Patients with toxic encephalopathy

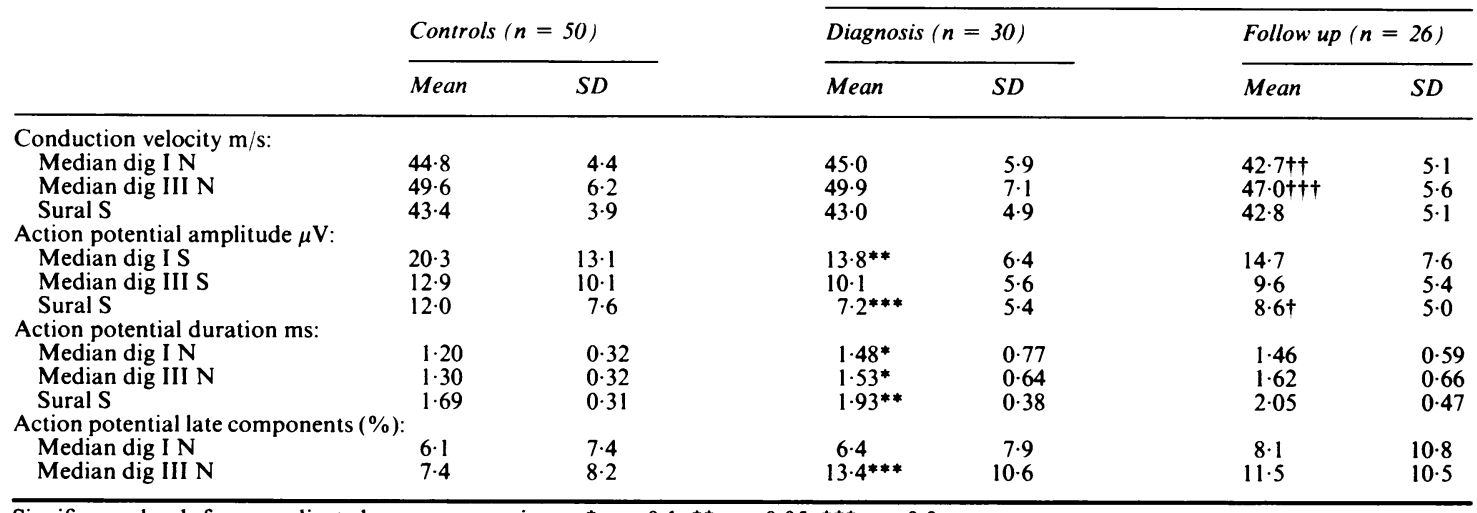

Significance levels for age adjusted group comparisons: ${ }^{*} p<0 \cdot 1,{ }^{* *} p<0.05, * * * p<0 \cdot 0$.

Significance levels in $t$ test for paired data in comparisons between the two examinations: $\dagger_{p}<0.1,+\dagger p<0.05,+\dagger+p<0.01$.

$\mathrm{N}$, needle electrodes; $\mathrm{S}$, surface electrodes. 


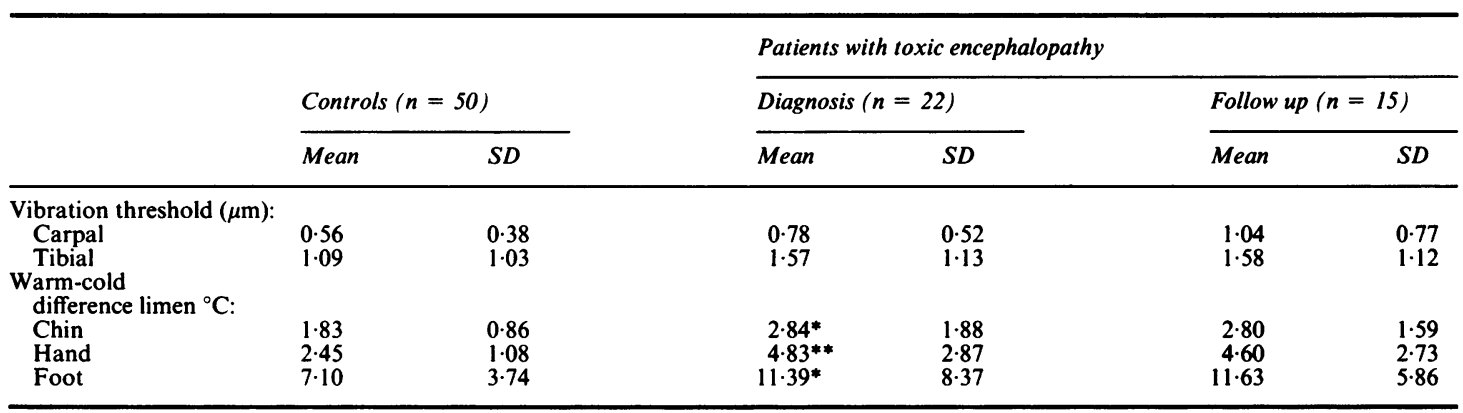

Significance levels for age adjusted group comparisons: ${ }^{*} p<0.05 ;{ }^{* *} p<0.001$.

No difference between the two examinations of the patient group was statistically significant.

sidered. We found no correlation with measures that had changed significantly during the follow up period.

Changes during follow up in the two subgroups young and old were considered for the measures in which the complete patient group differed statistically significantly from the control group (fig 1). Subgroups were also considered if the change during follow up was significant for the complete group (fig 2). As seen in fig 1 , improvements in sensory nerve action potential amplitude and percentage of late components were more pronounced in the younger subgroup. By contrast, the younger subjects had greater reductions in conduction velocities (fig 2).

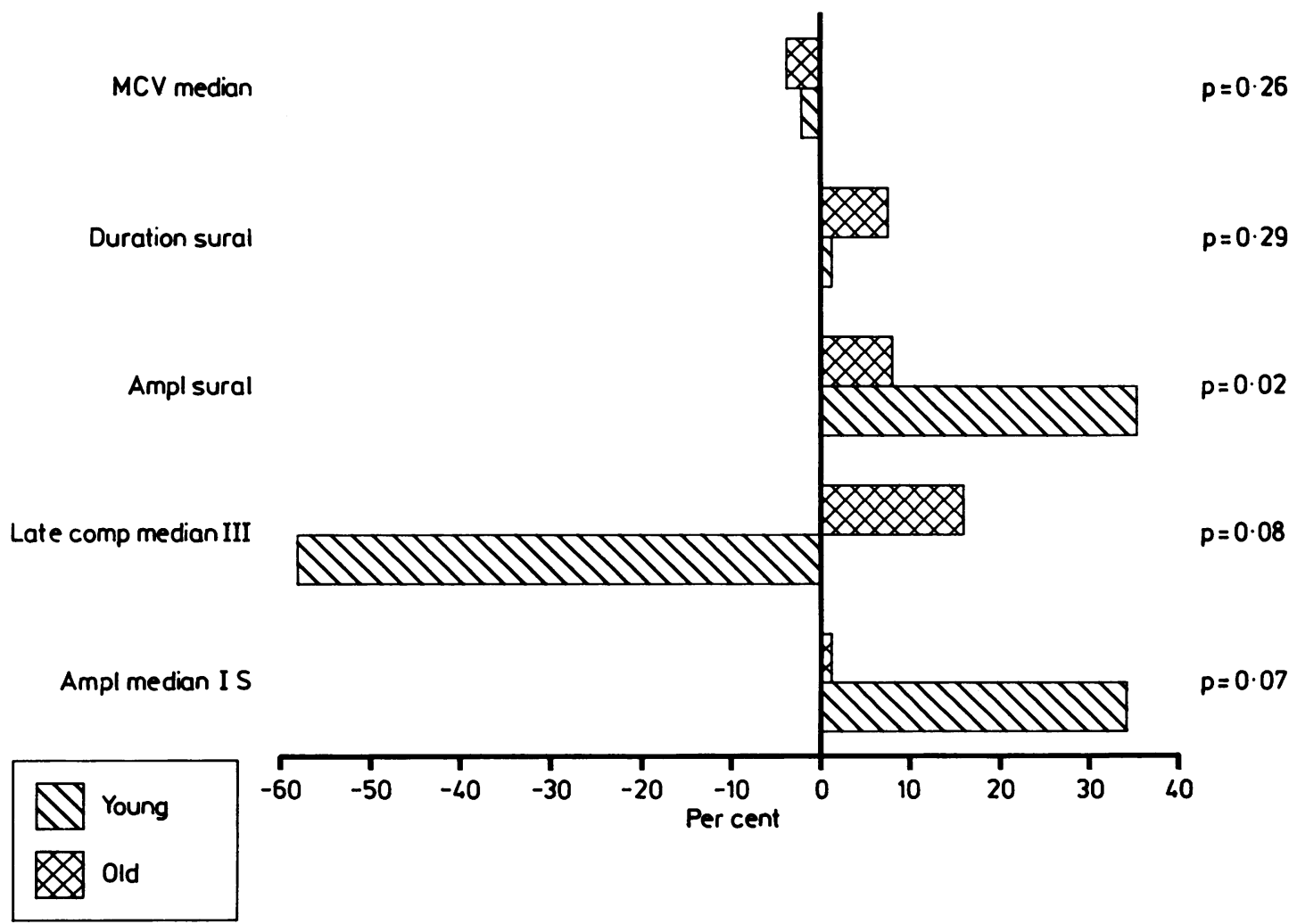

Fig 1 Relative changes during follow up of measures in which patients deviated significantly from controls. Subgroup young were aged 50 and under and subgroup old were over 50. Significance levels in Student's $t$ test for paired data in comparisons between two examinations in subgroups. 


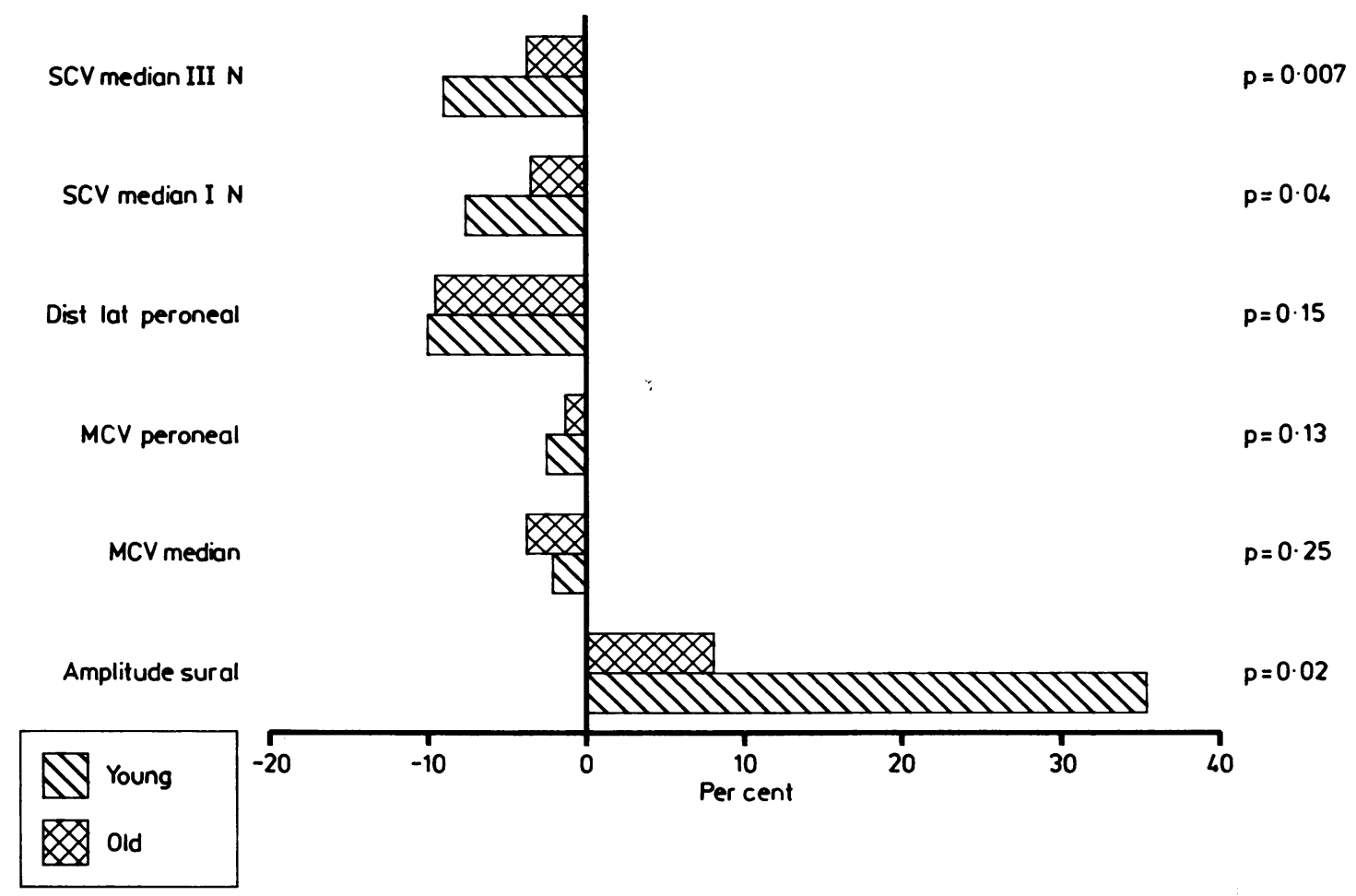

Fig 2 Relative changes during follow up of measures in which complete group deviated significantly from first examination. Subgroup young were aged 50 and under and subgroup old were over 50. Significance levels in Student's test for paired data in comparisons between two examinations in subgroups.

\section{Discussion}

Our results suggest that there is a mild axonal neuropathy in the sensory fibres of subjects with central nervous system dysfunction resulting from exposure to solvent mixtures. After cessation of exposure the sensory nerve function, as measured by nerve action potential amplitude, duration, and percentage of late components improved among the younger subjects in particular.

The neurographic changes were small from a clinical point of view. Signs and symptoms of sensory neuropathy, however, were found in $50 \%$ of the subjects. ${ }^{15 b}$ In this group interference from other diseases on the effects of solvents is unlikely. Our preliminary reports on the same group of patients were made without the present strict selection of subjects ${ }^{18}$ (and I Roseń et al, International Conference on Solvent Toxicity, Stockholm, 1984). Signs of axonal degeneration in the sensory fibres have been reported as the most sensitive indication of alcohol induced neuropathy. ${ }^{19}$ We did not find such changes in a group of alcohol abusers when age adjustment was made statistically in the group comparisons. ${ }^{18}$ Including the
11 subjects excluded due to their missing complete participation did not change the results.

Peripheral nerve dysfunction has previously been found in a high proportion of subjects with "chronic solvent intoxication." "Some evidence of solvent mixture induced peripheral dysfunction has been presented in several cross sectional studies of solvent exposed controls. ${ }^{4620}$ Sural nerve biopsy specimens from symptom free workers selected from the study of Elofsson et $a l^{5}$ disclosed only discreet changes. ${ }^{21}$ Exposed subjects differed from non-exposed by the existence of paranodal axonal mitochondria which contained glycogen like particles. ${ }^{22}$ The functional meaning of this observation is unknown.

It is difficult to explain the paradoxical reduction of the conduction velocities among the younger subjects in particular. They had improvements in the measurements of axonal function after cessation of exposure and the patient group did not differ from the controls at the first examination. If the age regression is used for estimating expected decrease the time between the two examinations is too short to be the reason. Exposure differences, except for longer exposure time in subgroup old, have to our knowledge 
not been present. The mean exposure free time was 40 months in both subgroups. This time interval is too long to explain a deterioration after the cessation of exposure as is found in hexacarbon induced neuropathy. ${ }^{10}$ We found no difference between the two subgroups in alcohol intake. Since the subjects have been examined intermixed during the observation period a systematic change in the equipment cannot be the reason. It cannot be ruled out that the subjects have been exposed to hexacarbons. If present, however, such exposure must have been slight.

One possibility is that peripheral nerve axons subjected to continuing exposure to solvents might show an increase in conduction velocity. Functional changes of the nodal membrane which had faded away at the follow up examination might be an explanation. An increase in conduction velocity has been caused by drugs $^{22}$ and the central conduction time is decreased with mild ischaemia in the central nervous system. ${ }^{23}$ It is not known whether similar phenomena can be elicited by solvents but this hypothesis gains some support from two cross sectional studies that show reduced action potentials together with some increase in conduction velocities in groups of exposed subjects. ${ }^{68}$ Further experimental research is needed for clarification.

The improvements during follow up in which the patient group had more pathological results than the control subjects is in accordance with the time course of the central nervous system function measured by EEG power spectrum analyses ${ }^{15}$ and regional cerebral blood flow. ${ }^{15 \mathrm{~s}}$

We conclude that exposure to mixtures of organic solvents may cause slight axonal degeneration in the sensory nerve fibres. The prognosis seems to be good, at least for younger subjects, if exposure is stopped.

We thank Professor Birgitta Hæger-Aronsen who introduced us to the problem and the subjects examined. The study was supported by grants from the Swedish Work Environment Fund (ASF 80-072 and 82-1011).

Requests for reprints to: Dr Palle Ørbæk, Yrkesmedicinska kliniken, Malmö allmänna sjukhus, S21401 Malmö.

\section{References}

1 Seppälainen AM, Haltia M. Carbon disulfide. In: Spencer PS, Schaumburg $\mathrm{HH}$, eds. Experimental and clinical neurotoxicology. Baltimore: Williams \& Wilkins, 1980:356-73.

2 Le Quesne PM. Acrylamide. In: Spencer PS, Schaumburg HH, eds. Experimental and clinical neurotoxicology. Baltimore: Williams \& Wilkins, 1980:309-25.

3 Spencer PS, Schaumburg HH. Organic solvent neurotoxicity. Facts and research needs. Scand $J$ Work Environ Health 1985;11(suppl 1):53-60.

4 Seppälainen AM, Husman K, Mårtenson C. Neurophysiological effects of long-term exposure to a mixture of organic solvents. Scand J Work Environ Health 1978:4:304-14.

5 Elofsson SA. Gamberale F. Hindmarsh T, et al. Exposure to organic solvents. A cross-sectional epidemiologic investigation on occupationally exposed industrial spray painters with special reference to the nervous system. Scand $J$ Work Environ Health 1980;6:239-73

6 Knave B, Anshelm Olson B, Elofsson S, et al. Long-term exposure to jet fuel. II. A cross-sectional epidemiologic investigation on occupationally exposed industrial workers with special reference to the nervous system. Scand J Work Environ Health 1978;4:19-45.

7 Rosén I, Haeger-Aronsen B, Rehnström S, Welinder H. Neurophysiological observations after chronic styrene exposure. Scand J Work Environ Health 1978:4(suppl 2):184-94.

8 Ørbæk P, Risberg J, Rosén I, et al. Effects of long-term exposure to organic solvents in the paint industry. A cross-sectional epidemiologic study with clinical and laboratory methods. Scand J Work Environ Health 1985:11(suppl 2):1-28.

9 Allen N, Mendell JR. Billmaier DJ. Fontaine RE. O'Neil J. Toxic polyneuropathy due to methyl n-butyl ketone. Arch Neurol 1975;32:209-18.

10 Cianchetti C, Abbritti G, Perticoni G. Siracusa A. Curradi F. Toxic polyneuropathy of shoe-industry workers. A study of 122 cases. J Neurol Neurosurg Psychiatry 1976;39:1151-61.

11 Seppälainen AM. Antti-Poika M. Time course of electrophysiological findings for patients with solvent poisoning. Scand J Work Environ Health 1983:9:15-24.

12 Le Quesne PM. Neurophysiological investigation of subclinical and minimal toxic neuropathies. Muscle Nerve 1978;1:392-5.

13 Shahani BT, Sumner AJ. Electrophysiological studies in peripheral neuropathy: early detection and monitoring. In: Ståhlberg E, Young RR, eds. Clinical neurophysiology. London: Butterworths, 1981:117-44

14 Ørbæk P, Rosén I. Svensson K. Power spectrum analysis of EEG at diagnosis and follow up of patients with solvent induced chronic toxic encephalopathy. Br J Ind Med (in press).

15 Ørbæk P. Lindgren M, Olivecrona H. Hæger-Aronsen B. Computed brain tomography and psychometric test performances in patients with solvent induced chronic toxic encephalopathy. $\mathrm{Br}$ J Ind Med 1987:44:175-9.

15a Hagstadius S, Ørbæk P, Risberg J, Lindgren M. Regional cerebral blood flow in organic solvent induced chronic toxic encephalopathy at the time of diagnosis and following cessation of exposure. Scand J Work Environ Health (in press).

15b Ørbæk P. Lindgren M. Prospective clinical and psychometric investigation of patients with solvent induced chronic toxic encephalopathy. Scand J Work Environ Health (in press).

16 Goldberg JM, Lindblom U. Standardized method of determining vibratory thresholds for diagnosis and screening in neurological investigation. J Neurol Neurosurg Psychiatry 1979;42:793-803.

17 Fruhstorfer $\mathrm{H}$, Lindblom U, Smith WG. Method for quantitative estimation of thermal thresholds in patients. J Neurol Neurosurg Psychiatry 1976;39:1071-5.

18 Rosen I. Neurophysiological aspects of organic solvent toxicity. Acta Neurol Scand 1984;70(suppl 100): 101-6.

19 D'Amour ML. Shahani BT, Young RR, Bird KT. The importance of studying sural nerve conduction and late response in evaluation of alcoholic subjects. Neurology 1979;29:1600-4.

20 Fagius J, Grönqvist B. Function of peripheral nerves and signs of polyneuropathy in solvent-exposed workers at a Swedish steelworks. Acta Neurol Scand 1978:57:305-16.

21 Berthold CH. Nordberg C. Hildebrand C, Conradi S, Sourander $\mathrm{P}$, Lugnegård $\mathrm{H}$. Sural nerve biopsies from workers with a history of chronic exposure to organic solvents and from normal control cases. Acta Neuropathol 1983;62:73-86.

22 Hopf HC. Drug-induced enhancement of conduction velocity. Abstract, 6th international congress of electromyography. Acta Neurol Scand 1979:60(suppl 73): 182 .

23 Branston NM, Ladds A, Symon L. Wang AD. Comparison of the effects of ischaemia on early components of the somatosensory evoked potential in brainstem, thalamus and cerebral cortex. $J$ Cereb Blood Flow Metab 1984;4:68-81. 